\title{
Article \\ The Diagnostic Role of Uric Acid to Creatinine Ratio for the Identification of Patients with Adverse Pulmonary Embolism Outcomes
}

\author{
Konstantinos Bartziokas *(D), Christos Kyriakopoulos (D), Dimitrios Potonos, Konstantinos Exarchos, \\ Athena Gogali and Konstantinos Kostikas (D)
}

check for

updates

Citation: Bartziokas, K.;

Kyriakopoulos, C.; Potonos, D.;

Exarchos, K.; Gogali, A.; Kostikas, K. The Diagnostic Role of Uric Acid to Creatinine Ratio for the Identification of Patients with Adverse Pulmonary Embolism Outcomes. Diagnostics 2022, 12, 193. https://doi.org/ 10.3390/diagnostics12010193

Academic Editor: Ludmilla Morozova-Roche

Received: 11 December 2021

Accepted: 11 January 2022

Published: 14 January 2022

Publisher's Note: MDPI stays neutral with regard to jurisdictional claims in published maps and institutional affiliations.

Copyright: (C) 2022 by the authors. Licensee MDPI, Basel, Switzerland. This article is an open access article distributed under the terms and conditions of the Creative Commons Attribution (CC BY) license (https:// creativecommons.org/licenses/by/ $4.0 /)$.
Respiratory Medicine Department, Faculty of Medicine, University of Ioannina, 45500 Ioannina, Greece; ckyriako123@gmail.com (C.K.); jimpotonos@yahoo.gr (D.P.); kexarcho@gmail.com (K.E.); athenagogali@yahoo.com (A.G.); ktkostikas@gmail.com (K.K.)

* Correspondence: bartziokas@gmail.com; Tel.: +30-69-3821-2878

\begin{abstract}
Background: Uric acid (UA) is the final product of purine metabolism and a marker of oxidative stress that may be involved in the pathophysiology of cardiovascular and thromboembolic disease. The aim of the current study is to investigate the potential value of UA to creatinine ratio $(\mathrm{UA} / \mathrm{Cr})$ as a diagnostic tool for the outcome of patients admitted with acute pulmonary embolism (PE) and the correlations with other parameters. Methods: We evaluated 116 patients who were admitted for PE in a respiratory medicine department. PE was confirmed with computed tomography pulmonary angiography. Outcomes evaluated were hospitalization duration, mortality or thrombolysis and a composite endpoint (defined as mortality or thrombolysis). Patients were assessed for PE severity with the PE Severity Index (PESI) and the European Society of Cardiology (ESC) 2019 risk stratification. Results: The median (interquartile range) UA/Cr level was 7.59 (6.3-9.3). UA/Cr was significantly associated with PESI $(p<0.001)$, simplified PESI $(p=0.019)$, and ESC 2019 risk stratification $(p<0.001$ ). The area under the curve (AUC) for prediction of 30-day mortality by UA/Cr was 0.793 (95\% CI: $0.667-0.918$ ). UA/Cr levels $\geq 7.64$ showed $87 \%$ specificity and $94 \%$ negative predictive value for mortality. In multivariable analysis UA/Cr was an independent predictor of mortality (HR (95\% CI): 1.620 (1.245-2.108), $p<0.001)$ and composite outcome (HR (95\% CI): 1.521 (1.211-1.908), $p<0.001)$. Patients with elevated UA/Cr levels $(\geq 7.64)$ had longer hospitalization (median (IQR) 7 (5-11) vs. $6(5-8)$ days, $p=0.006)$ ), higher mortality $(27.3 \%$ vs. $3.2 \%$, $p=0.001)$ and worse composite endpoint $(32.7 \%$ vs. $3.4 \%, p<0.001)$. Conclusion: Serum UA/Cr ratio levels at the time of PE diagnosis are associated with disease severity and risk stratification, and may be a useful biomarker for the identification of patients at risk of adverse outcomes.
\end{abstract}

Keywords: uric acid to creatinine ratio; pulmonary embolism; diagnosis; hospitalization; mortality; prognosis

\section{Introduction}

Pulmonary embolism (PE) covers a wide variety of clinical conditions, which range from asymptomatic, coincidentally revealed subsegmental thrombus found on chest CT scan to pressure-dependent PE complicated by multisystem organ failure and cardiogenic shock [1]. Pulmonary thromboembolism is a serious cardiovascular disease, causing a considerable level of morbidity and mortality. Hemodynamic status, concomitant comorbidities, and dysfunction of the right ventricle are predictors of short-term mortality. Indeed, 30-day mortality rates in patients stratified by the European Society of Cardiology (ESC) classification into high, intermediate-high, and intermediate-low risk groups were found to be $22 \%, 7.7 \%$, and $6.0 \%$, respectively [2]. Several clinical decision rules have been developed for the evaluation of the pretest clinical probability of PE [3], the most popular being the Well's rule [4] and the Geneva score [5], which has been revised in 2006 [6]. 
Oxidative stress upholds a crucial role in the pathophysiologic mechanism of various diseases [7]. Levels of uric acid (UA), the final oxidation product of purine metabolism, arise in conditions of impaired oxidative metabolism, namely cardiovascular disease, idiopathic pulmonary arterial hypertension, chronic thromboembolic pulmonary hypertension, metabolic syndrome, diabetes mellitus and obesity sleep apnea syndrome (OSAS) [8-13]. Hyperuricemia has been associated with increased mortality and is considered as an independent predictor of death in patients at high risk of cardiovascular disease [14]. High serum UA levels have long been known to indicate poor prognosis in certain acute diseases [15].

Previous studies have evaluated the role of serum uric acid to creatinine ratio $(\mathrm{UA} / \mathrm{Cr}$ ) and demonstrated the association with the presence of COPD, lung function, COPD severity, dyspnea, disease progression and prediction of exacerbation risk [16-18]. UA/Cr has also been associated with hypertension, metabolic syndrome, type 2 diabetes and malignancy $[17,19,20]$.

The aim of the present study was to investigate the potential diagnostic value of serum $\mathrm{UA} / \mathrm{Cr}$ in the identification of patients with worse prognosis, including adverse outcomes in patients admitted to hospital due an acute PE.

\section{Materials and Methods}

\subsection{Study Design}

Subjects objectively diagnosed with acute pulmonary thromboembolism, according to current guidelines, and those age $>18$ years, were included in this observational study between January 2019 and December 2020. The study was conducted at the Respiratory Medicine Department of University Hospital of Ioannina that serves as a referral center for patients with suspected pulmonary thromboembolism. This study was conducted in accordance with the amended Declaration of Helsinki and was approved by the Institutional Review Board (Approval number: 27947, approval date 15 October 2019). Informed consent was obtained from all individuals involved in the study. The study outcomes did not affect the future management of the patients, and the authors declare that the patient's personal data have been secured.

\subsection{Subjects and Setting}

The study inclusion criteria included symptomatic pulmonary thromboembolism confirmed by computed tomography pulmonary angiography, demographic data, troponin, systolic pressure and pulse on admission. Patients were excluded from the study if they met any of the following criteria: (1) referral to our hospital after the beginning of treatment; (2) end-stage renal failure, presence of sepsis, acute coronary syndromes, acute cerebrovascular disease, acute or chronic aortic dissection, acute or chronic infectious diseases, acute or inflammatory diseases such as acute myocarditis and/or pericarditis; (3) any conditions affecting lactic acid (LA) and blood gas analysis, such as severe anemia or metabolic acidosis; and (4) any medication use, at the time or during the month before the baseline examination, that might alter UA levels and/or LA metabolism, such as metformin, acetaminophen, and amoxicillin or consumption of great amount of alcohol.

Risk groups based on early mortality rate were stratified according to the 2019 ESC guidelines [1] and PESI and simplified PESI scores [21,22]. Patients were classified as being at high, intermediate-high, intermediate-low, or low risk, according to the ESC classification. Patients were classified by a numeric number and/or class from I to V (PESI alone) according to the PESI and simplified PESI. Risk adjusted therapy was administered based on these risk classifications. For the evaluation of the pretest clinical probability of PE, Wells rule [4] and the Geneva score [5] were calculated during the initial visit to the emergency department.

Thirty-day all-cause mortality was accepted as the primary end point based on inhospital deaths or civil registries, as well as duration of hospital stay. Adverse composite outcomes included mortality and clinical conditions that would result in death unless 
treated with following strategies recommended by the ESC guidelines [1]: (1) thrombolysis, (2) thrombectomy, or (3) extracorporeal membrane oxygenation (ECMO).

Treatment decisions were made by the physicians caring for the subject according to the current ESC guidelines and not influenced by the study protocol. All patients completed follow-up at one month after enrolment. Follow-up included one telephone interview and one surveillance face-to-face evaluation during the one month of study participation in the out-patient clinic.

\subsection{Biochemical Analysis}

Fasting venous blood samples were taken from an antecubital vein with minimal stasis on admission and were centrifuged within $15 \mathrm{~min}$ of collection. Troponin-T or hs Troponin-T levels were determined by a quantitative electrochemiluminescence assay (cut-off value $<0.010 \mathrm{ng} / \mathrm{mL}$ and cut-off value $0.014 \mathrm{ng} / \mathrm{mL}$, respectively). Serum $\mathrm{TnI}$ concentrations were performed on the ADVIA Centaur XP immunoassay analyzer (Siemens Healthcare Diagnostics Inc., Tarrytown, NY, USA). Plasma D-dimer levels were assessed using the BCS XP coagulation analyzer (Siemens Healthcare Diagnostics, Marburg, Germany). Serum UA was measured by enzymatic method (Olympus AU640; Hamburg, Germany) using an enzymatic spectrophotometric method, during the first diagnosis of pulmonary embolism. The normal range of serum uric acid levels was $2.4-6.0 \mathrm{mg} / \mathrm{dL}$ (females) and $4.4-7 \mathrm{mg} / \mathrm{dL}$ (males), and values are expressed as $\mathrm{mg} / \mathrm{dL}$ (milligrams per deciliter). The normal range of serum creatinine was between 0.6 and $1.2 \mathrm{mg} / \mathrm{dL}$. Blood cell enumeration and white blood cell differential counts were performed in an automated hematology analyzer (Sysmex K-4500, Roche Diagnostics, Kobe, Japan). All samples were analyzed within $2 \mathrm{~h}$ following blood collection without storage. Arterial LA levels were assessed by a sample of arterial blood gases (ABGs), during the initial visit to the emergency department. Values are expressed as mmol/L (millimoles per liter) for LA. ABGs samples were collected from the radial artery in heparinized syringes and gauged with auto-analyzer (ABL3000 auto-analyser Radiometer Co., Tokyo, Japan).

\subsection{Statistical Analysis}

The Kolmogorov-Smirnov and Shapiro-Wilk tests were used to assess conformity with a normal distribution. Categorical values were analyzed using the $\chi 2$ test or Fisher's exact test (chi-square test) as appropriate and were reported as $n$ (\%). Continuously measured variables were compared by Student's $t$-test when normally distributed or by the Mann-Whitney U ( 2 categories) and Kruskal-Wallis ( $>3$ categories) test for nonnormally distributed variables. Continuous parameters with normal distribution are reported as means $\pm \mathrm{SD}$, while those without normal distribution are reported as median (interquartile range). Correlations between the individual parameters were calculated using the Pearson or Spearman rank correlation coefficients $\left(\mathrm{r}_{\mathrm{s}}\right)$ as appropriate. Multivariable logistic regression analysis was performed to assess the relationship between independent variables and 30-day mortality, hospitalization days and adverse outcome. Prognostic factors with a $p$ value significance of $<0.05$ in the univariate analysis were entered in the multivariate model as a stepwise descending method. The level of statistical significance was set at $p$-value $<0.05$.All statistical analyses were performed using SPSS for Windows 26.0 (SPSS, Chicago, IL, USA) software and MedCalc version 12.

\section{Results}

We evaluated 168 consecutive patients admitted for PE and 116 (69.1\%) were eligible for inclusion in the present study. The flow chart of study participants is shown in Figure 1. The mean age \pm SD of the patients was $53.47 \pm 7.87$ years and $38.8 \%$ were males. The demographic characteristics of the 116 patients who were included in data analysis are presented in Table 1. Patients were divided into two groups according to the presence of serum uric acid to creatinine levels above or below the best value for mortality received through the receiver operating characteristic analysis $(\geq 7.636, n=55$ or $<7.636, n=61$, 
respectively). Age and gender distribution were the same in the two groups $(p=0.132$ and 1.000, respectively). Patients with higher serum $\mathrm{UA} / \mathrm{Cr}$ had higher respiratory rate $(p<0.001)$, higher PCO2 $(p<0.001)$, higher lactic acid $(p=0.002)$ and higher d-dimers $(p=0.013)$. Moreover, cardiopulmonary disease, defined as heart failure or chronic pulmonary disease, was more frequent in patients with higher UA/Cr levels; 8 (13\%) vs. 22 $(40 \%), p=0.004$.

\subsection{Association of UA/Cr with PESI Score, Simplified PESI and ESC Risk-Stratification}

Serum median (IQR) uric acid to creatinine ratio (UA/Cr) level was 7.59 (6.34-9.35). $\mathrm{UA} / \mathrm{Cr}$ levels were correlated with PESI score $(r=0.673, p<0.001$, Figure 2$)$. Serum UA/Cr levels were 6.35 (5.81-7.63) in low $(n=31,26.8 \%)$; and 8.00 (6.94-9.81) in high-risk group $(n=85,73.2 \%)$ based on simplified PESI risk-stratification $(p<0.001)$ (Table 1, Figure 3a).

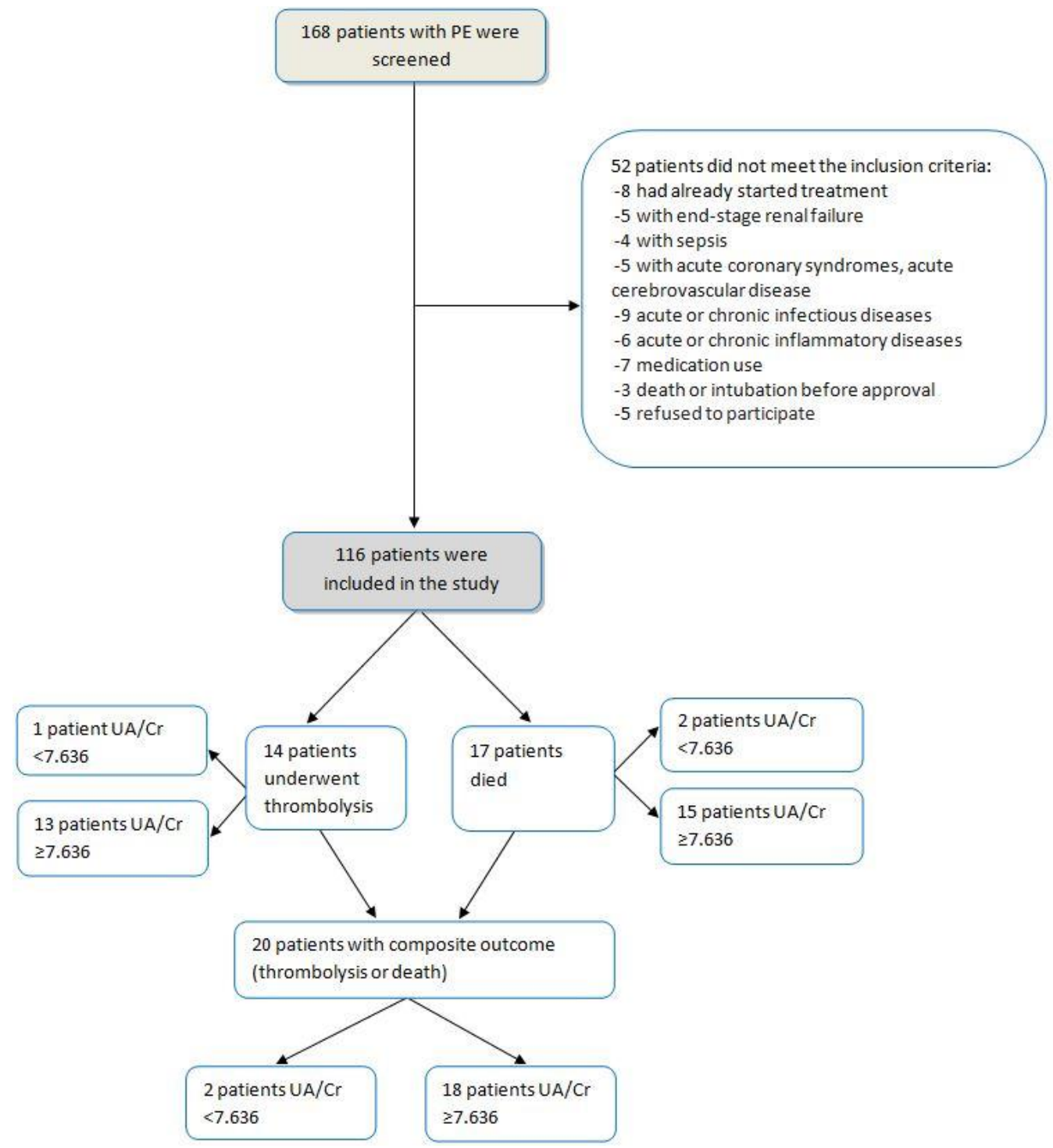

Figure 1. Flow chart of the study participants. 
Table 1. Baseline characteristics of patients with pulmonary embolism.

\begin{tabular}{|c|c|c|c|c|}
\hline Variables & $\begin{array}{c}\text { Total } \\
(n=116)\end{array}$ & $\begin{array}{c}\text { UA/Create }<7.636 \\
(n=61)\end{array}$ & $\begin{array}{c}\mathrm{UA} / \mathrm{Create} \geq 7.636 \\
(n=55)\end{array}$ & $p$ \\
\hline \multicolumn{5}{|l|}{ Demographic Factors } \\
\hline Age, mean $\pm S D$, years & $53.47 \pm 7.87$ & $54.48 \pm 7.32$ & $52.35 \pm 8.37$ & 0.132 \\
\hline Male sex, $n(\%)$ & $45(38.8)$ & $23(37.7)$ & $22(39.9)$ & 1.000 \\
\hline \multicolumn{5}{|l|}{ Clinical Parameters } \\
\hline SBP, median (IQR) & $135(120-140)$ & $137(128-146)$ & $135(120-140)$ & 0.091 \\
\hline Heart Rate, median (IQR) & $100(95-105)$ & $100(97-106)$ & $101(92-106)$ & 0.956 \\
\hline Respiratory Rate, median (IQR) & $28(21-31)$ & $21(18.5-23.5)$ & $31(30-32)$ & $<0.001$ \\
\hline \multicolumn{5}{|l|}{ Comorbidities, $n(\%)$} \\
\hline Malignancy & $27(23.2)$ & $11(18.1)$ & $16(29.1)$ & 0.190 \\
\hline Thrombophilia & $21(18.1)$ & $12(19.7)$ & $9(16.3)$ & 0.470 \\
\hline DVT symptoms & $29(25)$ & $17(27.8)$ & $12(21.8)$ & 0.286 \\
\hline Cardiopulmonary disease & $33(28.4)$ & $8(13.1)$ & $22(39.9)$ & 0.004 \\
\hline \multicolumn{5}{|l|}{ Laboratory Biomarkers, median (IQR) } \\
\hline $\mathrm{Ua} / \mathrm{Cr}$ & $7.59(6.34-9.35)$ & $6.40(5.61-7.12)$ & $9.5(8.12-10.61)$ & $<0.001$ \\
\hline $\mathrm{UA}, \mathrm{mg} / \mathrm{dL}$ & $6.8(6.4-7.87)$ & $6.5(6.1-6.9)$ & $7.7(6.9-8.5)$ & $<0.001$ \\
\hline D-dimers, ng/mL & $475(350-720)$ & $380(300-640)$ & $520(390-740)$ & 0.013 \\
\hline Oxygen saturation (\%) & $91(88-93)$ & $91(88-93)$ & $90(87-93)$ & 0.987 \\
\hline $\mathrm{PaO}_{2}, \mathrm{~mm} \mathrm{Hg}$ & $73(70-75)$ & $73(70-75.5)$ & $73(71.7-74.7)$ & 0.640 \\
\hline $\mathrm{PaCO}_{2}, \mathrm{~mm} \mathrm{Hg}$ & $36(33.4-38.6)$ & $34.3(32.1-36.4)$ & $37.9(34.8-38.2)$ & $<0.001$ \\
\hline Lactic acid, mmol/L & $1.73(1.72-1.77)$ & $1.73(1.72-1.74)$ & $1.74(1.73-1.78)$ & 0.002 \\
\hline $\mathrm{WBC} \times 10^{3} / \mu \mathrm{L}$ & $9.9(8.94-12.5)$ & $9.9(8.8-12.06)$ & $10.1(9-12.97)$ & 0.437 \\
\hline Platelets $\chi 10^{3} / \mu \mathrm{L}$ & $365(295-457)$ & $370(300-467)$ & $359(280-447)$ & 0.617 \\
\hline $\mathrm{CRP}, \mathrm{mg} / \mathrm{dL}$ & $68(12-100)$ & $54(11-87)$ & $78(14-138)$ & 0.180 \\
\hline $\mathrm{Na} \mathrm{mEq} / \mathrm{L}$ & $138(135-140)$ & $138(136-139)$ & $137(134-139)$ & 0.122 \\
\hline HSTPN, pg/mL & $20(14-40)$ & $31(14-45)$ & $17(14-29)$ & $<0.001$ \\
\hline \multicolumn{5}{|c|}{ Pulmonary Embolism Classification Tools } \\
\hline \multicolumn{5}{|l|}{ ESC 2019 algorithm } \\
\hline Low, $n(\%)$ & $18(15.5)$ & $17(27.9)$ & $1(1.8)$ & $<0.001$ \\
\hline Intermediate-low, $n(\%)$ & $55(47.4)$ & $39(63.9)$ & $16(29.1)$ & $<0.001$ \\
\hline Intermediate-high, $n(\%)$ & $35(30.2)$ & $5(8.2)$ & $30(54.5)$ & $<0.001$ \\
\hline High, $n(\%)$ & $8(6.9)$ & $0(0)$ & $8(13.8)$ & 0.002 \\
\hline PESI, median (IQR) & $113(70-144)$ & $76(62-88)$ & $144(126-161)$ & $<0.001$ \\
\hline sPESI, median (IQR) & $1(0-1)$ & $1(0-1)$ & $1(1-2)$ & 0.019 \\
\hline sPESI risk, high, $n(\%)$ & $85(73.2)$ & $37(60.6)$ & $48(87.2)$ & 0.001 \\
\hline
\end{tabular}

Bold indicates statistical significance. SBP: systolic blood pressure; DVT: deep vein thrombosis; Cardiopulmonary disease: chronic heart failure or pulmonary disease; UA/Cr: serum uric acid to creatinine ratio; UA: uric acid; $\mathrm{PaO}_{2}$ : arterial partial pressure of oxygen; $\mathrm{PaCO}_{2}$ : arterial partial pressure of carbon dioxide; WBC: white blood cells; CRP: c-reactive protein; HSTPN: high-sensitive troponin; ESC: European Society of Cardiology; PESI: pulmonary embolism severity index; sPESI: simplified PESI. 


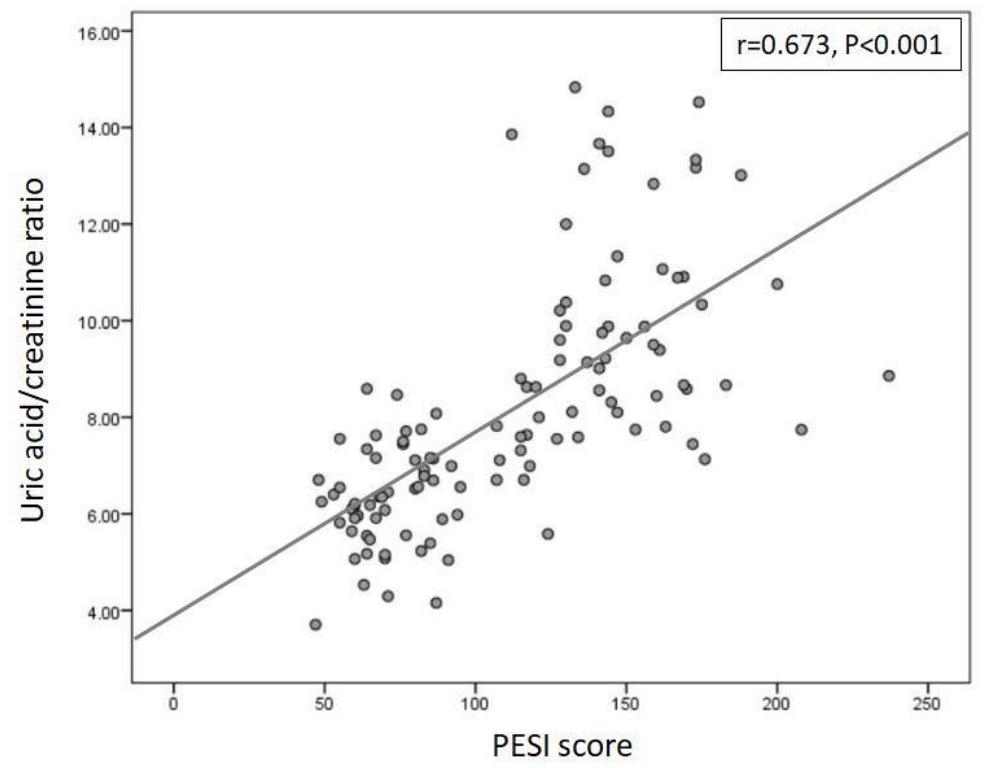

Figure 2. Correlation of uric acid to creatinine ratio levels with PESI score.

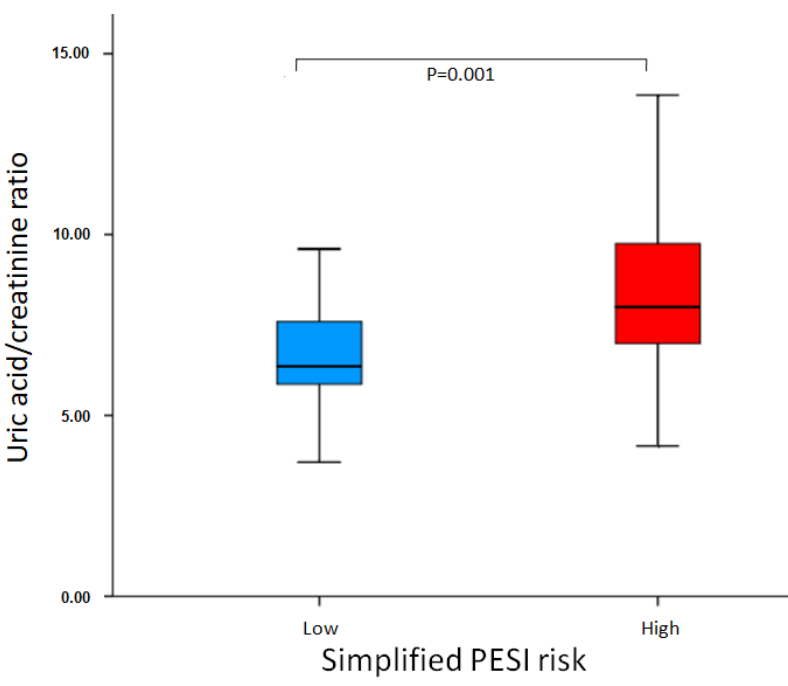

(a)

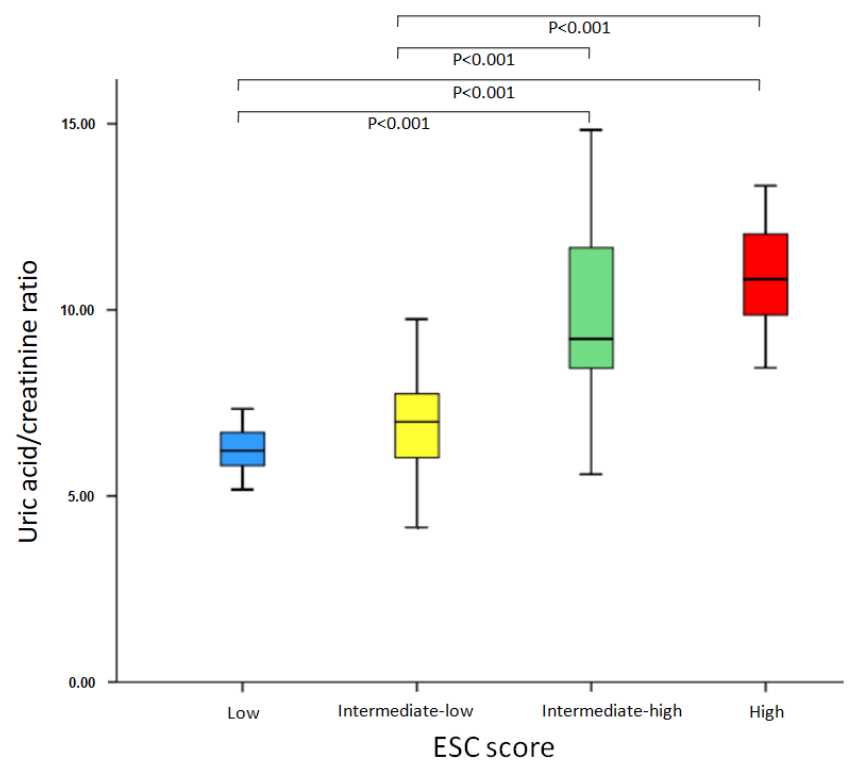

(b)

Figure 3. (a) Association of Uric acid to creatinine ratio levels with simplified PESI risk-stratification. (b) Association of Uric acid to creatinine ratio levels with simplified ESC 2019 risk-stratification.

Serum UA/Cr levels were $6.21(5.77-6.77)$ in low $(n=18,15.5 \%) ; 6.98$ (5.98-7.75) in intermediate-low $(n=55,47.4 \%) ; 9.22(8.31-12.00)$ in intermediate-high, $(n=35,30.2 \%)$; and $10.82(9.62-12.60)$ in high-risk group $(n=8,6.9 \%)$ based on 2019 ESC risk-stratification $(p<0.001)$ (Table 1, Figure 3b).

\subsection{Association of UA/Cr Levels with Hospitalization Outcome}

$\mathrm{UA} / \mathrm{Cr}$ levels were correlated with duration of hospitalization $(r=0.382, p<0.001)$. Patients with elevated UA/Cr levels $(\geq 7.636)$ had longer hospitalization (median (IQR) 7 (5-11) vs. $6(5-8)$ days, $p=0.006))$, higher mortality (\%) (27.3 vs. 3.2, $p=0.001)$ and worse composite endpoint (\%) (32.7 vs. 3.4, $p<0.001)$ (Table 2$)$. 
Table 2. Comparison of hospitalization outcomesin patients with low and high serum uric acid levels.

\begin{tabular}{|c|c|c|c|c|}
\hline & Total & UA/Create $<7.636$ & UA/Create $\geq 7.636$ & $p$ \\
\hline Subjects, $n$ & 116 & 61 & 55 & \\
\hline Hospitalization days, median (IQR) & $6(5-8)$ & $6(5.5-8.5)$ & $7(5-11)$ & 0.003 \\
\hline Mortality, $n(\%)$ & $17(14.6)$ & $2(3.2)$ & $15(27.3)$ & $<0.001$ \\
\hline Thrombolysis, $n(\%)$ & $14(12)$ & $1(1.6)$ & $13(23.6)$ & $<0.001$ \\
\hline Composite outcome, $n(\%)$ & $20(17.2)$ & $2(3.4)$ & $18(32.7)$ & $<0.001$ \\
\hline
\end{tabular}

Bold indicates statistical significance.

3.3. Associations of Demographic and Clinical Variables with Duration of Hospitalization, 30-Day Mortality and Adverse Composite Outcome

Multivariate linear regression analysis, as a stepwise descending method with the variables that were significant factors in the univariate analysis, revealed that duration of hospitalization was associated with elevated WBCs $(p<0.001)$, elevated platelets $(p=0.002)$ and higher UA/Cr $(p=0.003)$ (Table 3).

Table 3. Predictors of hospitalization days.

\begin{tabular}{|c|c|c|c|c|c|c|}
\hline \multirow[b]{2}{*}{ Variables } & \multicolumn{3}{|c|}{ Univariate } & \multicolumn{2}{|l|}{ Multivariate } & \multirow[b]{2}{*}{$p$} \\
\hline & HR $(95 \%$ CI) & Beta & $p$ & HR $(95 \%$ CI $)$ & Beta & \\
\hline Age & $0.002(-0.085-0.090)$ & 0.004 & 0.962 & & & \\
\hline Male & $1.135(-0.258-2.528)$ & 0.15 & 0.109 & & & \\
\hline Malignancy & $2.199(0.627-3.772)$ & 0.251 & 0.007 & $1.373(-0.416-3.162)$ & 0.157 & 0.131 \\
\hline RV dysfunction & $1.875(0.542-3.209)$ & 0.252 & 0.006 & $0.723(-1.100-2.546)$ & 0.097 & 0.433 \\
\hline $\begin{array}{c}\text { Cardiopulmonary } \\
\text { disease }\end{array}$ & $0.260(-1.261-1.780)$ & 0.032 & 0.736 & & & \\
\hline Heart rate & $-0.030(-0.114-0.055)$ & -0.065 & 0.490 & & & \\
\hline Respiratory rate & $0.108(-0.010-0.226)$ & 0.168 & 0.071 & $-0.154(-0.343-0.036)$ & -0.239 & 0.11 \\
\hline SBP & $-0.029(-0.069-0.010)$ & -0.136 & 0.144 & & & \\
\hline Oxygen saturation & $0.044(-0.105-0.194)$ & 0.055 & 0.558 & & & \\
\hline $\mathrm{PaCO}_{2}$ & $0.137(-0.091-0.365)$ & 0.111 & 0.237 & & & \\
\hline WBC & $0.001(0.000-0.001)$ & 0.412 & $<0.001$ & $0.001(0.000-0.001)$ & 0.35 & $<0.001$ \\
\hline $\mathrm{Na}$ & $-0.257(-0.503--0.012)$ & -0.191 & 0.04 & $0.103(-0.163-0.369)$ & 0.076 & 0.444 \\
\hline Hs TPNI & $-0.012(-0.052-0.027)$ & -0.057 & 0.545 & & & \\
\hline CRP & $0.008(0.000-0.015)$ & 0.192 & 0.039 & $0.003(-0.005-0.010)$ & 0.064 & 0.507 \\
\hline Platelets & $-0.006(-0.011--0.001)$ & -0.207 & 0.026 & $-0.001(0.000-0.000)$ & -0.258 & 0.002 \\
\hline UA/Creat & $0.574(0.316-0.831)$ & 0.382 & $<0.001$ & $0.598(0.209-0.987)$ & 0.398 & 0.003 \\
\hline PESI score & $0.024(0.008-0.039)$ & 0.275 & 0.003 & $0.006(-0.021-0.034)$ & 0.006 & 0.652 \\
\hline sPESI score & $0.971(-0.570-2.512)$ & 0.116 & 0.214 & & & \\
\hline ESC Risk Classification & $1.152(0.329-1.975)$ & 0.251 & 0.006 & $0.190(-1.042-1.421)$ & 0.041 & 0.761 \\
\hline
\end{tabular}

In univariate analyses, 30-day mortality correlated with malignancy $(p<0.001)$, thrombophilia $(p=0.036) \mathrm{RV}$ dysfunction $(p=0.012)$, higher respiratory rate $(p=0.005)$, higher $\mathrm{PCO}_{2}(p<0.001)$, higher lactic acid $(p=0.002)$, elevated WBCs $(p=0.002)$, hyponatremia $(p<0.001)$, higher CRP $(p<0.001)$, higher PESI $(p=0.001)$ and ESC score $(p<0.001)$, high sPESI risk $(p=0.036)$ and higher UA/Cr $(p<0.001)$. However, age, sex, platelets, low blood pressure, cardiopulmonary disease and heart rate did not affect 30-day mortality. 
Multivariate logistic regression analysis, as a stepwise descending method with the variables that were significant factors in the univariate analyses, demonstrated that 30-day mortality was associated with malignancy $(p=0.003)$, higher CRP $(p=0.007)$, and higher UA/Cr $(p<0.001)$ (Table 4).

Table 4. Predictors of 30-Day mortality.

\begin{tabular}{|c|c|c|c|c|c|c|}
\hline \multirow[b]{2}{*}{ Variables } & \multicolumn{3}{|c|}{ Univariate } & \multicolumn{3}{|c|}{ Multivariate } \\
\hline & HR $(95 \%$ CI $)$ & Beta & $p$ & HR $(95 \%$ CI $)$ & Beta & $p$ \\
\hline Age & $0.974(0.910-1.042)$ & 0.026 & 0.444 & & & \\
\hline Male & $0.890(0.312-2.536)$ & -0.117 & 0.827 & & & \\
\hline Malignancy & $0.145(0.048-0.435)$ & -1.930 & 0.001 & $0.110(0.026-0.470)$ & -2.206 & 0.003 \\
\hline RV dysfunction & $0.204(0.057-0.777)$ & -1.561 & 0.019 & $1.006(0.034-29.614)$ & 0.006 & 0.997 \\
\hline $\begin{array}{c}\text { Cardiopulmonary } \\
\text { disease }\end{array}$ & $1.346(0.405-4.476)$ & 0.297 & 0.627 & & & \\
\hline Heart rate & $1.008(0.946-1.074)$ & 0.008 & 0.806 & & & \\
\hline Respiratory rate & $1.166(1.038-1.311)$ & 0.154 & 0.010 & $0.873(0.679-1.123)$ & -0.136 & 0.291 \\
\hline SBP & $0.981(0.952-1.010)$ & -0.019 & 0.200 & & & \\
\hline Oxygen saturation & $0.990(0.888-1.103)$ & -0.011 & 0.849 & & & \\
\hline $\mathrm{PaCO}_{2}$ & 1.467 (1.158-1.858) & 0.383 & 0.001 & $1.172(0.859-1.597)$ & 0.158 & 0.316 \\
\hline WBC & $1.000(1.000-1.001)$ & 0.0003 & 0.004 & $1.000(1.000-1.001)$ & 0.001 & 0.222 \\
\hline $\mathrm{Na}$ & $0.658(0.528-0.821)$ & -0.418 & $<0.001$ & $1.122(0.766-1.642)$ & 0.115 & 0.555 \\
\hline HSTPNI & $1.002(0.973-1.032)$ & 0.002 & 0.894 & & & \\
\hline CRP & 1.008 (1.003-1.013) & 0.008 & 0.001 & 1.009 (1.002-1.015) & 0.009 & 0.007 \\
\hline Platelets & $1.000(1.000-1.001)$ & 0.001 & 0.120 & & & \\
\hline $\mathrm{UA} / \mathrm{Cr}$ & 1.575 (1.264-1.963) & 0.454 & $<0.001$ & $1.620(1.245-2.108)$ & 0.482 & $<0.001$ \\
\hline PESI score & $1.020(1.006-1.033)$ & 0.020 & 0.003 & $0.999(0.965-1.034)$ & -0.001 & 0.951 \\
\hline sPESI score & $1.577(0.865-2.874)$ & 0.455 & 0.137 & & & \\
\hline ESC Risk Classification & $3.559(1.696-7.469)$ & 1.269 & 0.001 & $2.215(0.510-9.618)$ & 0.795 & 0.289 \\
\hline
\end{tabular}

Bold indicates statistical significance. RV: right ventricular; Cardiopulmonary disease: chronic heart failure or pulmonary disease; SBP: systolic blood pressure; $\mathrm{PaCO}_{2}$ : arterial partial pressure of carbon dioxide; WBC: white blood cells; HSTPN: high-sensitive troponin; CRP: c-reactive protein; UA/Cr: serum uric acid to creatinine ratio; PESI: pulmonary embolism severity index; sPESI: simplified PESI; ESC: European Society of Cardiology.

In univariate analyses, adverse composite outcome correlated with malignancy $(p=0.002)$, thrombophilia $(p=0.021)$, RV dysfunction $(p=0.002)$, higher respiratory rate $(p=0.002)$, higher PCO2 $(p<0.001)$, higher lactic acid $(p=0.001)$, elevated WBCs $(p=0.010)$, hyponatremia $(p<0.001)$, higher CRP $(p<0.001)$, higher PESI $(p=0.001)$ and ESC score $(p<0.001)$, high sPESI risk $(p=0.016)$ and higher UA/Cr $(p<0.001)$ However, age, sex, platelets, low blood pressure, cardiopulmonary disease and heart ratedid not affect composite outcome.

Multivariate logistic regression analysis, as a stepwise descending method, with the variables that were significant factors in the univariate analyses highlighted that composite outcome was associated with malignancy $(p=0.014)$, higher CRP $(p=0.013)$, and higher $\mathrm{UA} / \mathrm{Cr}(p=0.001)$ (Table 5). 
Table 5. Predictors of adverse composite outcome.

\begin{tabular}{|c|c|c|c|c|c|c|}
\hline \multirow[b]{2}{*}{ Variables } & \multicolumn{3}{|c|}{ Univariate } & \multicolumn{3}{|c|}{ Multivariate } \\
\hline & HR $(95 \%$ CI) & Beta & $p$ & HR $(95 \%$ CI) & Beta & $p$ \\
\hline Age & $0.995(0.935-1.058)$ & -0.005 & 0.868 & & & \\
\hline Male & $0.733(0.277-1.940)$ & -0.310 & 0.532 & & & \\
\hline Malignancy & $0.215(0.078-0.597)$ & -1.536 & 0.003 & $0.214(0.062-0.735)$ & -1.540 & 0.014 \\
\hline RV dysfunction & $0.162(0.045-0.590)$ & -1.818 & 0.006 & 1.915 (0.162-22.627) & -0.650 & 0.606 \\
\hline $\begin{array}{c}\text { Cardiopulmonary } \\
\text { disease }\end{array}$ & $0.913(0.318-2.622)$ & -0.091 & 0.866 & & & \\
\hline Heart rate & $0.976(0.919-1.036)$ & -0.025 & 0.421 & & & \\
\hline Respiratory rate & 1.179 (1.054-1.317) & 0.164 & 0.004 & $0.926(0.770-1.112)$ & -0.077 & 0.411 \\
\hline SBP & $0.992(0.965-1.020)$ & -0.008 & 0.558 & & & \\
\hline Oxygen saturation & $1.013(0.908-1.131)$ & 0.013 & 0.811 & & & \\
\hline $\mathrm{PaCO}_{2}$ & $1.416(1.148-1.746)$ & 0.348 & 0.001 & $1.123(0.877-1.439)$ & 0.116 & 0.357 \\
\hline WBC & $1.000(1.000-1.001)$ & 0.001 & 0.012 & $1.000(0.998-1.002)$ & 0.001 & 0.178 \\
\hline $\mathrm{Na}$ & $0.703(0.577-0.856)$ & -0.353 & $<0.001$ & $1.067(0.790-1.844)$ & 0.065 & 0.673 \\
\hline HSTPNI & $1.015(0.988-1.042)$ & 0.014 & 0.283 & & & \\
\hline CRP & 1.007 (1.003-1.012) & 0.007 & 0.002 & 1.007 (1.001-1.012) & 0.007 & 0.013 \\
\hline Platelets & $1.000(1.000-1.000)$ & 0.000 & 0.199 & & & \\
\hline $\mathrm{UA} / \mathrm{Cr}$ & $1.537(1.248-1.894)$ & 0.430 & $<0.001$ & $1.521(1.211-1.908)$ & 0.419 & $<0.001$ \\
\hline PESI score & 1.022 (1.009-1.035) & 0.021 & 0.001 & 1.001 (0.973-1.030) & 0.001 & 0.951 \\
\hline sPESI score & $1.491(0.846-2.627)$ & 0.399 & 0.167 & & & \\
\hline ESC Risk Classification & $3.926(1.899-8.117)$ & 1.368 & $<0.001$ & $2.100(0.822-5.365)$ & 0.742 & 0.121 \\
\hline
\end{tabular}

Bold indicates statistical significance. RV: right ventricular; Cardiopulmonary disease: chronic heart failure or pulmonary disease; SBP: systolic blood pressure; $\mathrm{PaCO}_{2}$ : arterial partial pressure of carbon dioxide; WBC: white blood cells; HSTPN: high-sensitive troponin; CRP: c-reactive protein; UA/Cr: serum uric acid to creatinine ratio; PESI: pulmonary embolism severity index; sPESI: simplified PESI; ESC: European Society of Cardiology.

\subsection{Comparison with Existing Risk-Factor Prediction Models}

A level of $\mathrm{UA} / \mathrm{Cr} \geq 7.636$ showed $65 \%$ sensitivity, $87 \%$ specificity, $46 \%$ positive predictive value, $97 \%$ negative predictive value and $81 \%$ accuracy for 30 -day mortality. In receiver operating characteristic analysis the area under the curve was 0.793 for 30-day mortality $(95 \%$ CI $0.667-0.918, p<0.001)$ and 0.793 for the composite outcome (95\% CI $0.684-0.902$, $p<0.001$ ) (Figure 4). The performance of UA/Cr was similar with ESC Risk Classification for both 30-day mortality (AUC (95\% CI) $0.793(0.667-0.918)$ vs. $0.774(0.670-0.877)$ ) and composite outcome $(0.793(0.684-0.902)$ vs. $0.791(0.697-0.884))$, better than PESI score (0.734 (0.621-0.847) and $(0.753(0.651-0.855))$, respectively; while simplified PESI was not diagnostic $(0.622(0.496-0.748)$ and $(0.611(0.495-0.727))$, respectively (Table 6). 


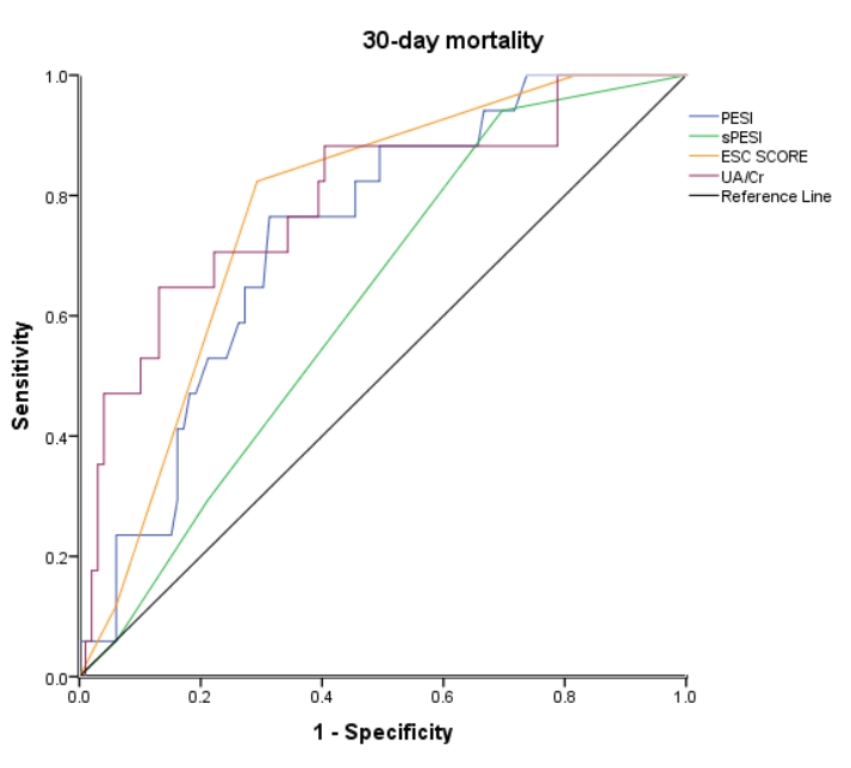

(a)

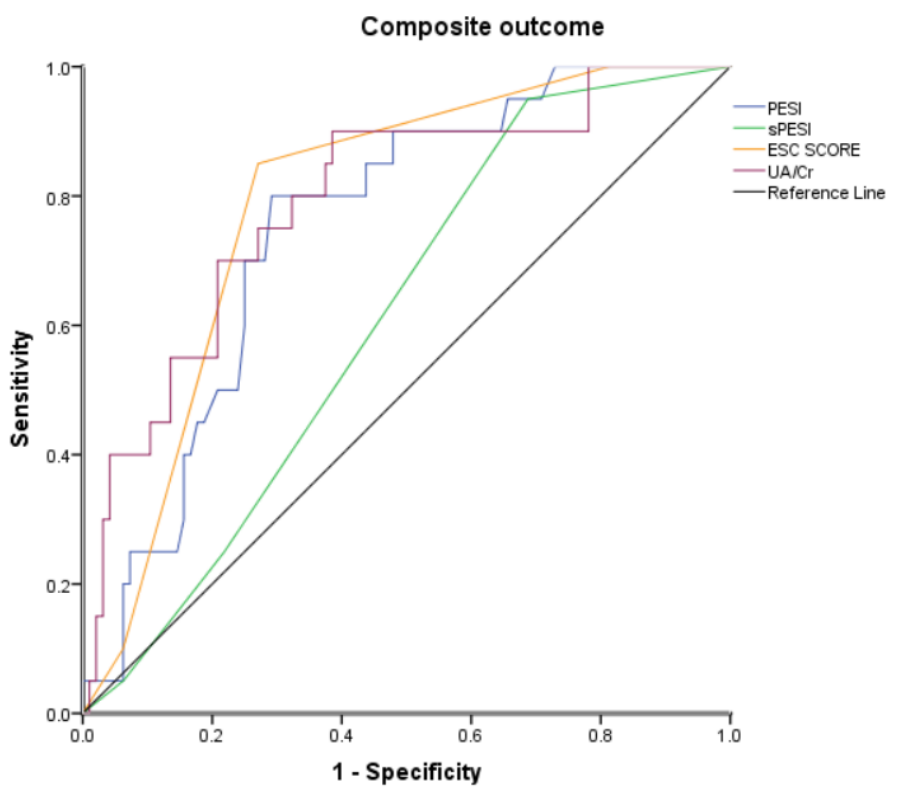

(b)

Figure 4. (a) ROC curves evaluating the diagnostic performance ofPESI, sPESI, ESC 2019 risk classification and uric acid to creatinine ratio as a predictor of 30-day mortality. (b) ROC curves evaluating the diagnostic performance ofPESI, sPESI, ESC 2019 risk classification and uric acid to creatinine ratio as a predictor of composite outcome.

Table 6. The performance of each risk factor prediction model with respect to predicting 30-day mortality and composite outcome.

\begin{tabular}{ccc}
\hline & 30-Day Mortality & Composite Outcome \\
\hline Predictors & AUC (95\% CI) & AUC (95\% CI) \\
\hline PESI score & $0.734(0.621-0.847)$ & $0.753(0.651-0.855)$ \\
PESI score, class I-V & $0.738(0.629-0.847)$ & $0.763(0.664-0.861)$ \\
sPESI score & $0.622(0.496-0.748)$ & $0.611(0.495-0.727)$ \\
sPESI score, class 0-1 & $0.622(0.495-0.749)$ & $0.631(0.513-0.749)$ \\
ESC 2019 Risk Classification & $0.774(0.670-0.877)$ & $0.791(0.697-0.884)$ \\
UA/Cr & $0.793(0.667-0.918)$ & $0.793(0.684-0.902)$
\end{tabular}

PESI: pulmonary embolism severity index; sPESI: simplified PESI; ESC: European Society of Cardiology; UA/Cr: serum uric acid to creatinine ratio.

\section{Discussion}

In this study, we found that high serum UA/Cr level in subjects with pulmonary thromboembolism is an independent predictor of mortality and composite outcome. Higher serum UA/Cr level was significantly associated with PESI, simplified PESI and the ESC 2019 risk stratification. Moreover, serum UA/Cr levels were positively correlated with disease severity, duration of hospitalization and clinical and laboratory parameters. Therefore, it may be postulated that serum UA/Cr levels may be considered as a prognostic marker for patients with pulmonary thromboembolism.

One of the first studies investigating the relationship between serum UA levels and the risk of thromboembolic events in subjects with non-valvular AF was conducted by Numa et al. [23]. In this study the risk of a thromboembolic event was increased by 1.45 times independent of serum creatinine level in subjects with a high serum UA level. Similar to our results, Babaoglu et al. [24], found the lowest and the highest UA levels in subjects with low- and high-risk pulmonary thromboembolism, respectively. Serum UA levels were statistically significant in subjects with intermediate- or high-risk pulmonary thromboembolism, compared with subjects with low-risk pulmonary thromboembolism. 
However, contrary to our study the relationship between serum UA level and mortality was not evident in this study.

Elevated UA levels have long been known to be a poor prognostic sign in acute illness $[25,26]$. Previous studies have demonstrated that elevated UA levels in pulmonary hypertension correlate with the severity of disease and were independently related to mortality on multivariate Cox proportional hazards analysis [10,27]. Kaplan-Meier survival curves demonstrated that subjects with high serum UA had a significantly higher mortality rate than those with low serum UA. Moreover, the mean UA levels were higher in subjects with chronic thromboembolic pulmonary hypertension [11].

In the present study higher UA/Cr levels were significantly associated with PESI, simplified PESI, and ESC 2019 risk stratification. There could be several reasons for this finding. Patients with pulmonary embolism and lower UA levels had less cardiopulmonary comorbidities compared to patients with higher UA levels, as is shown in Table 1, which are included in PESI and simplified PESI scoring system, and ESC 2019 risk stratification. Increased respiratory rate in these patients may also have contributed to this correlation. Additionally, 2019 ESC risk classification includes right ventricular function and cardiac biomarkers, which may better reflect hemodynamic significance that is related with increased serum uric acid levels.

In multivariable analysis UA/Cr was an independent predictor of mortality (30-day and all cause) and composite outcome. Previous studies have also reported that elevated uric acid was predictive of PE severity and mortality $[28,29]$. This finding may be explained by the fact that in our cohort, patients with pulmonary embolism and elevated serum $\mathrm{UA} / \mathrm{Cr}$ levels had increased $\mathrm{pCO}_{2}$, serum lactate, and d-dimers levels which may have had a significant impact on determining thrombolysis, severity of pulmonary embolism and oxygenation of the patients. UA, a byproduct of purine catabolism, is increased under the hypoxic conditions associated with diseases, such as obstructive sleep apnea, chronic obstructive pulmonary disease, and congestive heart failure $[13,30,31]$. In addition, UA levels have been shown to correlate negatively with cardiac output in patients with PE [28]. These findings suggest that hypoxia and decreased cardiac output in PE may elevate uric acid level, which correlates with the severity of PE and therefore with mortality and composite outcome.

Elevated serum UA level is associated with a wide range of diseases such as hypertension, chronic kidney disease, heart failure, and coronary artery disease, all these making it an important risk diagnostic tool [32]. Uric acid has been shown to be a useful biochemical marker of endothelial function, atherosclerosis, development of cardiovascular risk factors, and occurrence of cardiovascular events. It is worth mentioning that in patients with acute myocardial infarction, hyperuricemia is nowadays an established risk factor for adverse cardiovascular events, including cardiovascular mortality, independent of the presence of metabolic syndrome [33]. Recently there were established cut-off values for the optimal prediction of fatal myocardial infarction and all-cause mortality and increased serum UA values were able to independently predict the risk of premature acute myocardial infarction [34]. Finally, hyperuricemia is independently associated with an increased risk of both incident and recurrent atrial fibrillation. New evidence in this area was recently published [35] suggesting that not only serum UA levels above normal, but also a history of hyperuricemia contributes significantly to the risk of left atrial thrombosis.

To the best of our knowledge, there is no previous study evaluating the diagnostic role of $\mathrm{UA} / \mathrm{Cr}$ as a biomarker in the evaluation of pulmonary embolism outcome. One of the limitations of our study was that it was a single-center, tertiary-care hospital study with a certain number of participants. The results of the present study should be validated by larger prospective cohort studies, in order to confirm the utilization of $\mathrm{UA} / \mathrm{Cr}$ as a diagnostic tool during PE. A second limitation is that we assessed and followed-up the patients for a 30-day period after PE. Extended follow-up for a year could alter the results of our study. Thirdly, the evaluation of serum UA/Cr ratio was performed at a single moment in time. 


\section{Conclusions}

In the present study of patients admitted with $\mathrm{PE}$ we demonstrated that serum $\mathrm{UA} / \mathrm{Cr}$ ratio levels are associated with 30-day mortality, 30-day composite outcome, length of hospitalization, risk stratification, clinical and laboratory parameters and may be a useful biomarker for the identification of high-risk in patients at the time of the diagnosis of PE. Further prospective studies are needed for the evaluation of the diagnostic and prognostic role of this marker in larger populations with PE.

Author Contributions: Conceptualization: K.B., C.K. and K.K.; Data curation, K.B., C.K., K.E., A.G. and K.K.; Formal analysis, K.B., C.K., D.P., K.E. and K.K.; Investigation, K.B., C.K., D.P. and A.G.; Methodology, K.B.; Resources, A.G.; Software, C.K.; Validation, K.B.; Visualization, C.K.; Writingoriginal draft, K.B., C.K., D.P., K.E. and A.G.; Writing-review and editing, K.B. and K.K. All authors have read and agreed to the published version of the manuscript.

Funding: This research received no external funding.

Institutional Review Board Statement: This study was conducted in accordance with the amended Declaration of Helsinki and was approved by the Institutional Review Board of University Hospital of Ioannina (Approval number: 27947, approval date 15 October 2019).

Informed Consent Statement: Informed consent was obtained from all individuals involved in the study.

Data Availability Statement: The datasets generated during and/or analyzed during the current study are available from the corresponding author on reasonable request.

Conflicts of Interest: The authors declare no conflict of interest.

\section{References}

1. Konstantinides, S.V.; Meyer, G.; Becattini, C.; Bueno, H.; Geersing, G.-J.; Harjola, V.-P.; Huisman, M.V.; Humbert, M.; Jennings, C.S.; Jiménez, D.; et al. 2019 ESC Guidelines for the Diagnosis and Management of Acute Pulmonary Embolism Developed in Collaboration with the European Respiratory Society (ERS). Eur. Heart J. 2020, 41, 543-603. [CrossRef] [PubMed]

2. Becattini, C.; Agnelli, G.; Lankeit, M.; Masotti, L.; Pruszczyk, P.; Casazza, F.; Vanni, S.; Nitti, C.; Kamphuisen, P.; Vedovati, M.C.; et al. Acute Pulmonary Embolism: Mortality Prediction by the 2014 European Society of Cardiology Risk Stratification Model. Eur. Respir. J. 2016, 48, 780-786. [CrossRef] [PubMed]

3. Papaioannou, A.I.; Kostikas, K. Clinical Decision Rules for The Diagnostic Management of Suspected Acute Pulmonary Embolism: The Clinician ... Rules. Pneumon 2011, 24, 221-223.

4. Wells, P.S.; Anderson, D.R.; Rodger, M.; Stiell, I.; Dreyer, J.F.; Barnes, D.; Forgie, M.; Kovacs, G.; Ward, J.; Kovacs, M.J. Excluding Pulmonary Embolism at the Bedside without Diagnostic Imaging: Management of Patients with Suspected Pulmonary Embolism Presenting to the Emergency Department by Using a Simple Clinical Model and D-Dimer. Ann. Intern. Med. 2001, 135, 98-107. [CrossRef] [PubMed]

5. Wicki, J.; Perneger, T.V.; Junod, A.F.; Bounameaux, H.; Perrier, A. Assessing Clinical Probability of Pulmonary Embolism in the Emergency Ward: A Simple Score. Arch. Intern. Med. 2001, 161, 92-97. [CrossRef]

6. Le Gal, G.; Righini, M.; Roy, P.-M.; Sanchez, O.; Aujesky, D.; Bounameaux, H.; Perrier, A. Prediction of Pulmonary Embolism in the Emergency Department: The Revised Geneva Score. Ann. Intern. Med. 2006, 144, 165-171. [CrossRef]

7. Gospodaryov, D.V.; Lushchak, V. Oxidative stress: Cause and consequence of diseases. In Oxidative Stress and Diseases, 1st ed.; Lushchak, V., Ed.; InTech: Rijeka, Croatia, 2012; Volume 1, pp. 13-38.

8. Hisatome, I.; Li, P.; Miake, J.; Taufiq, F.; Mahati, E.; Maharani, N.; Utami, S.B.; Kuwabara, M.; Bahrudin, U.; Ninomiya, H. Uric Acid as a Risk Factor for Chronic Kidney Disease and Cardiovascular Disease-Japanese Guideline on the Management of Asymptomatic Hyperuricemia. Circ. J. 2020, 85, 130-138. [CrossRef]

9. Lindman, B.R.; Dávila-Román, V.G.; Mann, D.L.; McNulty, S.; Semigran, M.J.; Lewis, G.D.; de las Fuentes, L.; Joseph, S.M.; Vader, J.; Hernandez, A.F.; et al. Cardiovascular Phenotype in HFpEF Patients with or without Diabetes: A RELAX Trial Ancillary Study. J. Am. Coll. Cardiol. 2014, 64, 541-549. [CrossRef]

10. Bendayan, D.; Shitrit, D.; Ygla, M.; Huerta, M.; Fink, G.; Kramer, M.R. Hyperuricemia as a Prognostic Factor in Pulmonary Arterial Hypertension. Respir. Med. 2003, 97, 130-133. [CrossRef]

11. Korkmaz, A.; Ozlu, T.; Ozsu, S.; Kazaz, Z.; Bulbul, Y. Long-Term Outcomes in Acute Pulmonary Thromboembolism: The Incidence of Chronic Thromboembolic Pulmonary Hypertension and Associated Risk Factors. Clin. Appl. Thromb. Hemost. 2012, 18, 281-288. [CrossRef]

12. Ford, E.S.; Li, C.; Cook, S.; Choi, H.K. Serum Concentrations of Uric Acid and the Metabolic Syndrome among US Children and Adolescents. Circulation 2007, 115, 2526-2532. [CrossRef] [PubMed] 
13. Bartziokas, K.; Papaioannou, A.I.; Haniotou, A.; Nena, E.; Kostikas, K.; Steiropoulos, P. Serum Uric Acid and Arterial Lactate Levels in Patients with Obstructive Sleep Apnea Syndrome: The Effect of CPAP Treatment. Postgrad. Med. 2021, 133, 518-524. [CrossRef] [PubMed]

14. Ioachimescu, A.G.; Brennan, D.M.; Hoar, B.M.; Hazen, S.L.; Hoogwerf, B.J. Serum Uric Acid Is an Independent Predictor of All-Cause Mortality in Patients at High Risk of Cardiovascular Disease: A Preventive Cardiology Information System (PreCIS) Database Cohort Study. Arthritis Rheum. 2008, 58, 623-630. [CrossRef]

15. Warwick, G.; Thomas, P.S.; Yates, D.H. Biomarkers in Pulmonary Hypertension. Eur. Respir. J. 2008, 32, 503-512. [CrossRef]

16. Garcia-Pachon, E.; Padilla-Navas, I.; Shum, C. Serum Uric Acid to Creatinine Ratio in Patients with Chronic Obstructive Pulmonary Disease. Lung 2007, 185, 21-24. [CrossRef]

17. DurmusKocak, N.; Sasak, G.; Aka Akturk, U.; Akgun, M.; Boga, S.; Sengul, A.; Gungor, S.; Arinc, S. Serum Uric Acid Levels and Uric Acid/Creatinine Ratios in Stable Chronic Obstructive Pulmonary Disease (COPD) Patients: Are These Parameters Efficient Predictors of Patients at Risk for Exacerbation and/or Severity of Disease? Med. Sci. Monit. 2016, 22, 4169-4176. [CrossRef]

18. Rumora, L.; Hlapčić, I.; Popović-Grle, S.; Rako, I.; Rogić, D.; Čepelak, I. Uric Acid and Uric Acid to Creatinine Ratio in the Assessment of Chronic Obstructive Pulmonary Disease: Potential Biomarkers in Multicomponent Models Comprising IL-1beta. PLoS ONE 2020, 15, e0234363. [CrossRef]

19. Tao, J.; Shen, X.; Li, J.; Cha, E.; Gu, P.P.; Liu, J.; Zhu, W.; He, L.L.; Li, G.Q.; Wang, Z. Serum Uric Acid to Creatinine Ratio and Metabolic Syndrome in Postmenopausal Chinese Women. Medicine 2020, 99, e19959. [CrossRef]

20. Li, M.; Gu, L.; Yang, J.; Lou, Q. Serum Uric Acid to Creatinine Ratio Correlates with $\beta$-Cell Function in Type 2 Diabetes. Diabetes. Metab. Res. Rev. 2018, 34, e3001. [CrossRef] [PubMed]

21. Aujesky, D.; Obrosky, D.S.; Stone, R.A.; Auble, T.E.; Perrier, A.; Cornuz, J.; Roy, P.-M.; Fine, M.J. Derivation and Validation of a Prognostic Model for Pulmonary Embolism. Am. J. Respir. Crit. Care Med. 2005, 172, 1041-1046. [CrossRef]

22. Jiménez, D.; Aujesky, D.; Moores, L.; Gómez, V.; Lobo, J.L.; Uresandi, F.; Otero, R.; Monreal, M.; Muriel, A.; Yusen, R.D. Simplification of the Pulmonary Embolism Severity Index for Prognostication in Patients with Acute Symptomatic Pulmonary Embolism. Arch. Intern. Med. 2010, 170, 1383-1389. [CrossRef]

23. Numa, S.; Hirai, T.; Nakagawa, K.; Ohara, K.; Fukuda, N.; Nozawa, T.; Inoue, H. Hyperuricemia and Transesophageal Echocardiographic Thromboembolic Risk in Patients with Atrial Fibrillation at Clinically Low-Intermediate Risk. Circ. J. 2014, 78, 1600-1605. [CrossRef]

24. Babaoglu, E.; Hasanoglu, H.C.; Senturk, A.; Karalezli, A.; Kilic, H.; Aykun, G.; Oztuna, D. Importance of Biomarkers in Risk Stratification of Pulmonary Thromboembolism Patients. J. Investig. Med. 2014, 62, 328-331. [CrossRef]

25. Akbar, S.R.; Long, D.M.; Hussain, K.; Alhajhusain, A.; Ahmed, U.S.; Iqbal, H.I.; Ali, A.W.; Leonard, R.; Dalton, C. Hyperuricemia: An Early Marker for Severity of Illness in Sepsis. Int. J. Nephrol. 2015, 2015, 301021. [CrossRef] [PubMed]

26. Woolliscroft, J.O.; Colfer, H.; Fox, I.H. Hyperuricemia in Acute Illness: A Poor Prognostic Sign. Am. J. Med. 1982, 72, 58-62. [CrossRef]

27. Voelkel, M.A.; Wynne, K.M.; Badesch, D.B.; Groves, B.M.; Voelkel, N.F. Hyperuricemia in Severe Pulmonary Hypertension. Chest 2000, 117, 19-24. [CrossRef]

28. Shimizu, Y.; Nagaya, N.; Satoh, T.; Uematsu, M.; Kyotani, S.; Sakamaki, F.; Nakanishi, N.; Miyatake, K. Serum Uric Acid Level Increases in Proportion to the Severity of Pulmonary Thromboembolism. Circ. J. 2002, 66, 571-575. [CrossRef] [PubMed]

29. Ozsu, S.; Çoşar, A.M.; Aksoy, H.B.; Bülbül, Y.; Oztuna, F.; Karahan, S.C.; Ozlu, T. Prognostic Value of Uric Acid for Pulmonary Thromboembolism. Respir. Care 2017, 62, 1091-1096. [CrossRef]

30. Bartziokas, K.; Papaioannou, A.I.; Loukides, S.; Papadopoulos, A.; Haniotou, A.; Papiris, S.; Kostikas, K. Serum Uric Acid as a Predictor of Mortality and Future Exacerbations of COPD. Eur. Respir. J. 2014, 43, 43-53. [CrossRef]

31. Hare, J.M.; Johnson, R.J. Uric Acid Predicts Clinical Outcomes in Heart Failure: Insights Regarding the Role of Xanthine Oxidase and Uric Acid in Disease Pathophysiology. Circulation 2003, 107, 1951-1953. [CrossRef]

32. TTăpoi, L.; Șalaru, D.L.; Sascău, R.; Stătescu, C. Uric Acid-An Emergent Risk Marker for Thrombosis? J. Clin. Med. Res. 2021, 10, 1951-1953. [CrossRef]

33. Pugliese, N.R.; Mengozzi, A.; Virdis, A.; Casiglia, E.; Tikhonoff, V.; Cicero, A.F.G.; Ungar, A.; Rivasi, G.; Salvetti, M.; Barbagallo, C.M.; et al. The Importance of Including Uric Acid in the Definition of Metabolic Syndrome When Assessing the Mortality Risk. Clin. Res. Cardiol. 2021, 110, 1073-1082. [CrossRef]

34. Casiglia, E.; Tikhonoff, V.; Virdis, A.; Masi, S.; Barbagallo, C.M.; Bombelli, M.; Bruno, B.; Cicero, A.F.G.; Cirillo, M.; Cirillo, P.; et al Serum Uric Acid and Fatal Myocardial Infarction: Detection of Prognostic Cut-off Values: The URRAH (Uric Acid Right for Heart Health) Study. J. Hypertens. 2020, 38, 412-419. [CrossRef]

35. Zhang, X.; Hu, M.; Wang, X.; Zhang, C.; Chen, W.; Chen, S.; Zhou, J.; Chen, Y.; Lou, L.; Chen, G.; et al. New Perspective on the Risk Markers for Left Atrial Thrombosis in Patients with Atrial Fibrillation. Eur. J. Prev. Cardiol. 2020, 28, 641-647. [CrossRef] 